\title{
Optical Delay Amplified by Chirped Fiber Bragg Gratings
}

\author{
Byeong $\mathrm{Ha} \mathrm{Lee}{ }^{*}$ and Gopinath Mudhana \\ Department of Information and Communications, Kwangju Institute of Science and Technology, \\ Gwangju 500-712, KOREA
}

(Received October 2, 2003)

\begin{abstract}
We report a novel optical delay line that can be implemented using only optical fiber and fiber devices without the need for any bulk-optic devices such as lens, prism, and moving mirror. The dispersive property of a chirped fiber Bragg grating (CFBG) is exploited to get the delay. The proposed delay line constitutes two identical CFBGs cascaded in the reverse order with one of them being strained. Analysis reveals that the small displacement or the strain applied on the CFBG is effectively amplified in the delay line by the ratio of the minimum resonant wavelength and the reflection bandwidth of the CFBG. The dispersion properties of the CFBG with and without the strain are analyzed in detail. The theoretical performance of the proposed delay line is also discussed. Applications of the proposed delay line are expected in the field of high-speed optical coherence tomograpy.

OCIS codes : $170.4500,170.3880,050.1590,060.2310,230.1150$.
\end{abstract}

\section{INTRODUCTION}

An optical delay line which is the main part of any scanning interferometer has been developed mainly to monitor or to record the events that occur in an ultra-short time scale. For example, an autocorrelator composed of an optical delay line is used to measure the temporal width of an ultra-short optical pulse [1]. Where, a part of the input pulse is delayed and then combined with the other part of the pulse. By adjusting the delay length, the overlap between the two parts of the pulse can be adjusted. Thus, the temporal pulse width can be obtained by measuring the efficiency of the second harmonic generation (SHG) in a crystal, which has the maximum when both the signal and the delayed signal are overlapped completely.

Recently, optical delay lines are widely used in the field of Optical Coherence Tomography (OCT), which gives the bisectional image of a sample without dissection [2]. The delay line is used to get the depth information of the sample in the OCT. For that case, a fast delay line is preferred since the scanning speed of the delay line determines the image acquisition speed of the system. To get video-rate imaging (several frames per second), scanning speeds of more than a $\mathrm{kHz}$ are required. Furthermore, for the practical application of the $\mathrm{OCT}$, an optical fiber type delay line is preferred due to the compactness and other advantages of all fiber-based systems.

In this work, we propose a novel all fiber delay line that can be used for implementing a high speed OCT system. The in-fiber delay is obtained without the need for any bulk-optic components such as a collimating lens or a moving mirror by exploiting the distributed reflection characteristic of a chirped fiber Bragg grating (CFBG) [3]. The proposed delay line consists of two identical CFBGs cascaded in the reverse order with one of the CFBGs being strained to obtain the required delay. In this paper, we briefly review the concepts and problems of the typical delay lines used in OCT systems. Then we present the basic idea and the theoretical performance of the proposed delay line.

\section{OPTICAL DELAY LINE}

Conventional optical delay lines are fabricated usually based on bulk-optics. The simplest method is using a moving mirror, in which a collimated light beam is reflected by a reflector, such as a mirror or a prism that is fixed on a translation stage. The delay is adjusted by moving the reflector along with the translation stage. However, the scanning speed of the delay is limited by the speed of the translation stage, which cannot be more than few tens of $\mathrm{Hz}$ due to the weights of the bulk optic mirror and the stage itself. 
In order to obtain a higher scanning speed of the delay line, several methods have been proposed. One of the most powerful methods is using a rotating cube [4]. Light incident through a glass cube is reflected from the internal facets of the cube, and the beam emerging from the cube is reflected back with a fixed mirror. Because the total traveling length of the beam depends on the relative angle of the cube against the incident angle, the optical delay is obtained by rotating the cube. In this scheme, even though the cube is rather heavy, since the cube is rotating in one direction (not oscillating), high speed scanning becomes possible. Further, due to the symmetry of the cube one complete rotation $\left(360^{\circ}\right)$ gives 4 scannings. However, a rather big cube is used since the maximum delay length is proportional to the size of the cube. In order to get a delay length of several millimeters, a centimeter-order size cube should be used. Furthermore, the delay length is not linearly related to the rotating angle.

The other widely used technique is the Fourierdomain method that uses a diffraction grating, a focusing lens and a vibrating mirror (galvano-mirror) [5]. At first, a collimated incident beam is spectrally dispersed by a diffraction grating, and focused on a vibrating mirror with a lens. The lens focuses it in a line on the vibrating mirror according to its wavelength components. The vibrating mirror imposes a linear phase ramp on the spectrum and redirects the light back through the path identical to the input path. In this scheme, the group delay becomes proportional to the focal length of the lens and the rotating angle of the vibrating angle. Therefore, a small displacement of the vibrating mirror can be amplified to give a longer delay length. Since the required tilt angle $\left(<0.5^{\circ}\right)$ of the vibrating mirror is small, up to $4 \mathrm{kHz}$ scanning speed was reported by using a resonant galvano-mirror [5].

The fast scanning delay lines described above have good performance but are based on bulk-optic components such as lenses, cubes, diffraction gratings, and moving mirrors. Therefore, the delay system becomes bulky and has severe problems in optical alignment. Further, the obtained delay is not linearly proportional to the applied movement and might be polarization dependent.

An all-fiber delay line will overcome the problems of alignment and polarization dependency and introduce other merits such as compactness and cost effectiveness. The simplest method of the all-fiber delay line is stretching the fiber, which extends the physical length of the fiber to obtain the delay. However, the procurable delay is not long enough for the OCT system with optical fiber of a few tens of centimeters. To get a longer delay length, the fiber should be wound around a piezoelectric drum [6]. However, the winding induces polarization dependency and the scanning speed is limited by the big capacitance of the piezoelectric drum. Therefore, it is preferred to have a delay line that is composed of a short-length optical fiber but can provide the delay length of an order of $\mathrm{mm}$.

\section{CHIRPED FIBER BRAGG GRATINGS}

A fiber Bragg grating (FBG) is a fiber device that has a phase grating along the core of an optical fiber. The phase grating can be easily imprinted in the fiber by illuminating it with an ultraviolet beam through a phase mask. When the grating has a uniform periodicity, the beam propagating in the core of the fiber is reflected by the grating when its wavelength fulfills the Bragg or resonant condition of the grating [7]. The resonant wavelength is equal to the optical path length of the beam that takes a round trip between the adjacent grating elements:

$$
\lambda=2 \Lambda n
$$

where, $\Lambda$ is the periodicity of the grating and $n$ is the effective index of the core mode of the fiber. The wavelength $\lambda$ is called the Bragg or resonant wavelength of the grating.

A chirped fiber Bragg grating (CFBG) is the FBG whose periodicity is chirped. Fig. 1 depicts the CFBG whose periodicity is linearly increased along the length $z$ of the device [8]. Therefore, a shorter wavelength component $\lambda_{s}$ of the incident beam is reflected earlier than the one having a longer wavelength $\lambda_{l}$. Denoting the periodicity of the CFBG, at the input end $z=z_{s}$, as $\Lambda_{s}$, the periodicity of the CFBG with a chirping rate $\beta$ can be expressed as,

$$
\Lambda(z)=\Lambda_{s}+\beta\left(z-z_{s}\right)
$$

Since the periodicity in a CFBG is a function of the position, the resonant wavelength of the CFBG becomes a function of the position along the device also,

$$
\lambda(z)=2 n\left(\Lambda_{s}+\beta\left(z-z_{s}\right)\right)
$$

or it can be written as

$$
\lambda(z)=2 n \beta\left(z-z_{s}\right)+2 n \Lambda_{s}
$$

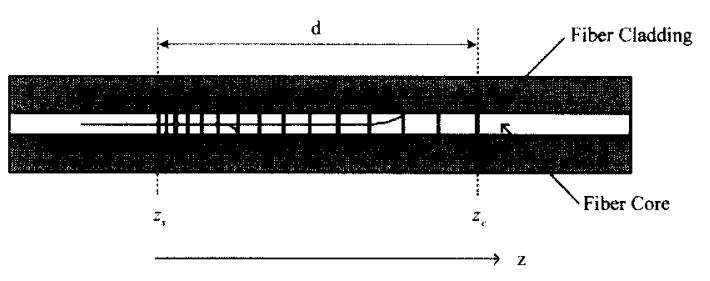

FIG. 1. Schematic of a chirped fiber Bragg grating. The grating has a length of $d$, and starts at $z=z_{s}$ and ends at $z=z_{e}$. The corresponding resonant wavelengths are $\lambda_{s}$ and $\lambda_{e}$, respectively. 


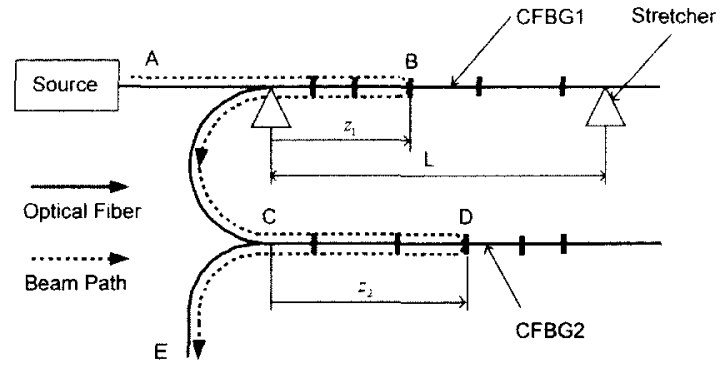

FIG. 2. Schematic of dispersion cancellation of CFBGs. Two identical CFBGs are cascaded in the reverse order. The dispersion from the CFBG1 is cancelled by the CFBG2. The fiber stretcher will be used to implement the proposed all-fiber delay line.

When we assume a positive chirping rate $\beta$, the CFBG reflects the shorter wavelength component of the beam having a broadband spectrum earlier than the longer one. That means the CFBG has a dispersion that depends on the chirping rate. When the chirping rate is negative or the direction of the incident beam is reversed, the CFBG gives a negative dispersion.

If we assume a device that is composed of two identical CFBGs cascaded in the reverse order as shown in Fig. 2, the incident beam reflects at $z_{1}$ according to the Bragg condition in the first CFBG taking the path $\mathrm{AB}$ followed by reflection at $z_{2}$ in the second CFBG. Thus, the total path length of the beam within both CFBGs is given by $2\left(z_{1}+z_{2}\right)$, where 2 is multiplied due to the round trip. Because both CFBGs are identical and reversely ordered, the total path length of the beam becomes constant or wavelength independent. The fiber stretcher in Fig. 2 will be used to apply strain on one of the CFBGs to get wavelength independent delay. To keep generality, the length of the strain-applied region of the fiber piece, $L$, is assu-

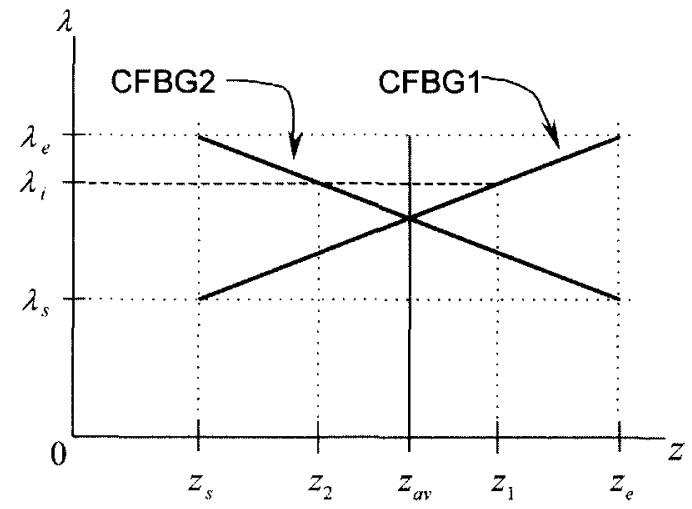

FIG. 3. Dispersion curves explaining the operation of Fig. 2. A beam having a wavelength $\lambda_{i}$ is reflected at $z_{1}$ of the CFBG1 and then at $z_{2}$ of the CFBG2. The average distance is the same regardless of the wavelength of the incident beam. med longer than the length of the CFBG, $d$, imprinted along the fiber, which will be discussed in the following sections.

As mentioned, the dispersion originated by the first CFBG is compensated by the second identical CFBG cascaded in the reverse direction. This dispersion compensation mechanism can be simply explained by drawing the dispersion curves of both CFBGs together as shown in Fig 3. In the figure, the vertical solid line, passing through the cross point of two curves, depicts the average of the path lengths of the beam in both CFBGs, $z_{a v}=\left(z_{1}+z_{2}\right) / 2$. The total path length of the beam in the grating pair is $2\left(z_{1}+z_{2}\right)$, as mentioned, which is equal to $4 z_{a v}$. Since $z_{a v}$ is wavelength independent so is the total path length. However, the dispersion curves of Fig. 3 correspond to linearly chirped ideal CFBGs. A practical CFBG has dispersion ripples called as the group delay ripple [9].

\section{STRAIN ON CHIRPED FIBER BRAGG GRATINGS}

When a CFBG is strained, the beam that would have reflected at position $z$ of the CFBG will be reflected at another position $z^{\prime}$. The difference between $z^{\prime}$ and $z$ or the shift in the reflection position will be proportional to the applied strain. The proportional coefficient is determined by the CFBG parameters. When a fiber is strained, the distance between any two points marked on the fiber is extended by the same rate. Thus, when a piece of fiber is strained from length $L$ to $L+a$, the applied strain $\varepsilon$ is defined as

$$
\varepsilon \equiv \frac{a}{L}
$$

The elongation ratio $\alpha$ defined as the ratio of the lengths of the fiber under strain and without strain is given as,

$$
\alpha \equiv \frac{L+a}{L}=1+\varepsilon
$$

Thus, the periodicity of the strained CFBG, $\Lambda^{\prime}(z)$ can be calculated from Eq. (2) in terms of the strain parameters $\alpha$ and $\varepsilon$ as,

$$
\Lambda^{\prime}(z)=\alpha \Lambda_{s}+\beta\left(z-\alpha z_{s}\right)
$$

Comparing with Eq. (2), we can see that $\Lambda_{s}$ and $z_{s}$ are increased by the same rate $\alpha$ because these are distances. While, $\beta$ and $z$ are not changed since $\beta$ is a ratio and $z$ is just a coordinate variable. Inserting Eq. (6) into the Bragg condition, Eq. (1), gives the 
wavelength of the beam reflected at a point $z^{\prime}$ of the strained CFBG as

$$
\lambda\left(z^{\prime}\right)=2 n^{\prime} \beta\left(z^{\prime}-\alpha z_{s}\right)+2 \alpha n^{\prime} \Lambda_{s}
$$

Comparing with Eq. (3), the dispersion curve in the coordinate frame of Fig. 3 is shifted linearly by the rate $\alpha$ and the dispersion slope is changed due to the change in the effective index of the strained-fiber because of the photo-elastic effect. In general, the refractive index of a material is decreased with strain due to the photo-elastic effect [10] as

$$
n^{\prime}=n\left(1-p_{e} \varepsilon\right)
$$

where, $p_{e}$ is the photo-elastic coefficient of the fiber.

\section{ALL FIBER OPTICAL DELAY LINE}

The slope of the dispersion curves given by Eqs. (3) and (7) are different from each other when plotted with respect to the physical length along the fiber. However, these slopes are the same when plotted with the optical path length (OPL) of the beam. The OPL is defined as the product between the physical length of the waveguide and the effective refractive index of the propagation mode. Therefore, $n z$ of Eq. (3) and $n^{\prime} z^{\prime}$ of Eq. (7) are the OPLs of the normal (without strain) and strained CFBGs, respectively. It means, in terms of the OPL, the resonant wavelength of the strained CFBG varies at the same rate with the case of no strain.

In Fig. 4., the dispersion curves of the normal and strained CFBGs are co-plotted as functions of the OPL. The figure shows that the dispersion curve is only shifted without change in the slope. The direction of the shift is along the line connecting the origin of the coordinate axes and the starting point of the

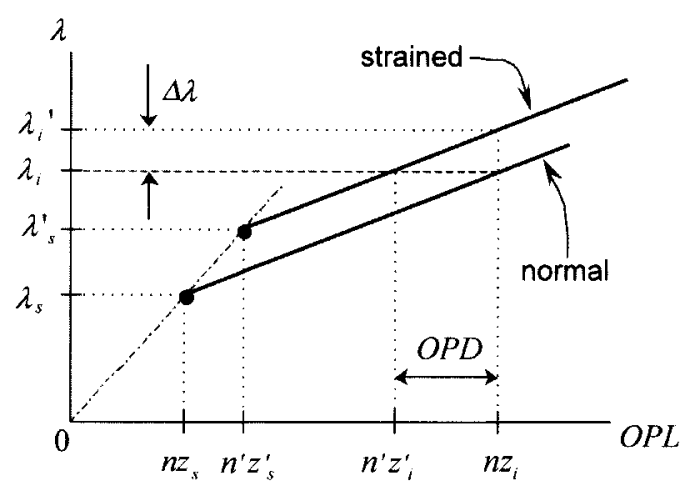

FIG. 4. Dispersion curves of the normal (without strain) and strained CFBGs co-plotted as functions of the optical path length (OPL). With strain, the dispersion curve is only shifted without change in the slope. The direction of the shift is along the line connecting the origin of the coordinate with the starting point of the grating, $\left(n z_{s}, \lambda_{s}\right)$. grating, $\left(n z_{s}, \lambda_{s}\right)$. From Eqs. (3) and 7, the difference of the resonant wavelengths of the normal and the strained CFBGs measured at the same point in the OPL frame, is calculated as

$$
\Delta \lambda=-2 \beta\left(n^{\prime} \alpha-n\right)\left(z_{s}-\frac{\Lambda_{s}}{\beta}\right)
$$

We note that the value of Eq. (9) is positive with a positive chirping rate $\beta>0$ and positive strain $\alpha>1$. Similarly, a beam having a certain wavelength $\lambda_{i}$ is reflected earlier from $n z_{i}$ to $n^{\prime} z_{i}^{\prime}$, when the strain is applied. By using the slope of the dispersion curve $2 \beta$, the gain in the OPL, or the optical path length difference (OPD) induced by the strain is expressed as

$$
O P D \equiv 2\left(n^{\prime} z_{i}^{\prime}-n z_{i}\right)=2\left(n^{\prime} \alpha-n\right)\left(z_{s}-\frac{\Lambda_{s}}{\beta}\right)
$$

where the factor of 2 was due to the round trip. Interestingly, the OPD does not depend on the wavelength of the incident beam.

Therefore, when two identical CFBGs are cascaded in the reverse order as shown in Fig. 2, and one of the CFBGs, CFBG1 for example, is strained, the total OPL is decreased by the amount described by Eq. (10). We had an OPL of a single CFBG as linear with respect to wavelength. However, by cascading with an identical CFBG in the reverse order, the dispersion can be eliminated, so that we can obtain wavelength independent OPD. By utilizing these concepts, an all-fiber optical delay line can be implemented. In other words, wavelength independent OPL delay by the amount described with Eq. (10) can be obtained by applying strain on any one of the CFBGs configured as shown in Fig. 2.

The proposed delay line has all the natural merits of the fiber-optic based system such as compactness, robustness, and alignment free operation. It is also free from polarization problems as the circular symmetry of a fiber is well preserved even with the gratings in the fiber. We require only small piezo-electric transducers since the fiber has small diameter. Finally, the scanning speed of the delay can be increased by utilizing a fast piezo-electric transducer.

\section{DISCUSSION}

From the knowledge that a fiber can not undergo bigger than a few percents strain, Eq. (10) can be simplified. From Eqs. (5) and (8), we have

$$
\begin{aligned}
n^{\prime} \alpha-n & =n\left(1-p_{e} \varepsilon\right)(1+\varepsilon)-n \\
& =n\left(1-p_{e}\right) \varepsilon-n p_{e} \varepsilon^{2}
\end{aligned}
$$


The last term of the equation can be neglected since the applicable strain is not too big. Therefore, Eq. (10) can be approximated as,

$$
O P D \approx 2 n\left(1-p_{e}\right) \varepsilon\left(z_{s}-\frac{\Lambda_{s}}{\beta}\right)
$$

It is more informative to express Eq. (12) with the resonant wavelengths of the CFBG because the chirping rate $\beta$ is not an easily measurable parameter. When the CFBG without strain has a length $d$ and a reflection bandwidth $\Delta \lambda$ from $\lambda_{s}$ to $\lambda_{e}$, the chirping rate $\beta$ is calculated from Eqs. (2) and (1) as

$$
\begin{aligned}
\beta & =\frac{1}{2 n} \frac{\Delta \lambda}{d} \\
\text { where } \quad \Delta \lambda & \equiv \lambda_{e}-\lambda_{s}
\end{aligned}
$$

Inserting these equations into Eq. (12) and using Eq. (1) we have

$$
O P D=2 n\left(1-p_{e}\right) \varepsilon\left(z_{s}-\frac{\lambda_{s}}{\Delta \lambda} d\right)
$$

For a particular case where the length of the fiber $L$ suffering strain is the same as the length of the grating $d$, Eq. (14) is further simplified as

$$
O P D=-2 n\left(1-p_{e}\right) a \frac{\lambda_{s}}{\Delta \lambda}
$$

where, we have used $z_{s}=0$ and Eq. (4). The parameter $a$ in the equation is the increase in the length of the fiber due to the applied strain. The term $2 n\left(1-p_{e}\right) a$ indicates the delay obtained by the strain-induced fiber length increment and the associated decrease in the fiber modal index. While, the ratio of the start wavelength and the reflection bandwidth of the CFBGs is the amount of amplification obtained by the proposed cascaded-CFBG configuration for the delay line. The amplification factor $\gamma$ is defined as

$$
\gamma \equiv \frac{\lambda_{s}}{\Delta \lambda}
$$

In Eq. (15), the negative sign appears because stretching the CFBG1 decreases the OPD. However, the sign can be made positive by stretching CFBG2 instead of CFBG1.

\section{CONCLUSION}

We have presented a novel all fiber optical delay line and discussed its properties. It is composed of two identical chirped fiber Bragg gratings (CFBGs) cascaded in the reverse order to cancel out the dispersions to each other. The wavelength independent delay is obtained by stretching one of the CFBGs.
The induced-strain shifts the dispersion curve of the CFBG but without changing its slope. Therefore, wavelength independent optical delay can be obtained from the CFBG pair though they are dispersive by nature. The strain-induced displacement applied on one of the CFBG is amplified by the proposed scheme and provides an amplified delay. The amplification factor is proportional to the ratio between the minimum resonant wavelength and the reflection bandwidth of the CFBG.

Since, the proposed delay line is based on optical fibers, it has many merits over the conventional bulkoptic-based delay lines such as being compact, robust, and alignment-free. It is also free from polarizationrelated problems due to the circular symmetry of the fiber and the fiber gratings. High-speed operation is expected because a high-speed piezo-electric transducer can be used for stretching a thin fiber.

\section{ACKNOWLEDGEMENTS}

This work was supported in part by the Korea Science and Engineering Foundation (KOSEF) through the Ultrafast Fiber-Optic Networks Research Center at Kwangju Institute of Science and Technology, by the Korean Ministry of Education through the BK-21 Project, and by the Ministry of Commerce, Industry and Energy (MOCIE) through the Industrial Base Funding projects.

${ }^{*}$ Corresponding author : leebh@kjist.ac.kr.

\section{REFERENCES}

[1] H. P. Weber, "Method for pulsewidth measurement of ultrashort light pulses generated by phase-locked lasers using nonlinear optics," J. Appl. Phys., vol. 38, pp. 2231-2234, 1967.

[2] D. Huang, E. A. Swanson, C. P. Lin, J. S. Schuman, W. G. Stinson, W. Chang, M. R. Hee, T. Flotte, K. Gregory, C. A. Puliafito, and J. G. Fujimoto, "Optical coherence tomography," Science, vol. 254, pp. 11781181, 1991.

[3] B. H. Lee, T.-J. Eom, E. Choi, Y.-J. Kim, and C. Lee, "All fiber delay line for OCT based on fiber gratings," in Asian Symposium on Biomedical Optics and Photomedicine 2002, TB2-1, pp. 140-141, 2002.

[4] J. Ballif, R. Gianotti, Ph. Chavanne, R. Walti, and R. P. Salathe, "Rapid and scalable scans at $21 \mathrm{~m} / \mathrm{s}$ in optical low-coherence reflectometry," Opt. Lett., vol. 22, no. 11, pp. 757-759, 1997.

[5] A. M. Rollins, M. D. Kulkarni, S. Yazdanfar, R. U.arunyawee, and J. A. Izatt, "In vivo video rate optical coherence tomography," Opt. Express, vol. 3, no.6, pp. 219-229, 1998. 
[6] G. J. Tearney, B. E. Bouma, S. A. Boppart, B. Golubovic, E. A. Swanson, and J. G. Fusimoto, "Rapid acquisition of in vivo biological images by use of optical coherence tomography," Opt. Lett., vol. 21, no. 17, pp. 1408-1410, 1996.

[7] T. Erdogan, "Fiber grating spectra," J. Light. Wave Technol., vol. 15, no. 8, pp. 1277-1294, 1997.

[8] K. C. Byron, K. Sugden, T. Bircheno, and I. Bennion, "Fabrication of chirped Bragg gratings in pho- tosensitive fibre," Electron. Lett., vol. 29, no. 18, pp. 1659-1660, 1993.

[9] R. Kashyap and M. L. Rocha, "On the group delay characteristics of chirped fibre Bragg gratings," Optics Communications, vol. 153, no. 6, pp. 19-22, 1998.

[10] W. W. Morey, J. R. Dunphy, and G. Meltz, "Multiplexing fiber Bragg grating sensors," Distributed and Multiplexed Fiber Optic Sensors, Proc. SPIE., vol. 1586, pp. 216-224, 1991. 